\title{
Adenovirus-directed Expression of Dominant Negative Estrogen Receptor Induces Apoptosis in Breast Cancer Cells and Regression of Tumors in Nude Mice
}

\author{
Eun Jig Lee, ${ }^{1}$ Monika Jakacka, ${ }^{1}$ W. Rachel Duan, ${ }^{1}$ Pei Yu Chien, ${ }^{1}$ Fred Martinson, ${ }^{1}$ Barry D. Gehm,,${ }^{1,2}$ \\ and J. Larry Jameson ${ }^{1,2}$ \\ ${ }^{1}$ Division of Endocrinology, Metabolism, and Molecular Medicine \\ ${ }^{2}$ Robert H. Lurie Comprehensive Cancer Center, Northwestern University Medical School, Chicago, IL, USA \\ Accepted October 12, 2001
}

\begin{abstract}
Background: Estrogen receptors (ER) are expressed in about two thirds of human breast cancer, and are an important pharmacological target for treatment of these tumors. Dominant negative forms of the ER have been suggested as an alternative method to disrupt ER function. In this study, we examined the effect of dominant negative ER mutants (ER 1-536 and L540Q) on ER-positive breast cancer cells in vitro and in vivo.

Materials and Methods: ER-positive T47D breast cancer cells were infected with adenoviral vectors expressing ER 1-536 and L540Q to examine the effects of the mutants on gene expression and cell growth. Adenoviral vectors containing the wild type ER (AdwtER) and $\beta$-galactosidase gene (AdGal) were used as controls.

Results: Ad1-536 or AdL540Q infection inhibited T47D cell growth and induced apoptosis, increasing Bax protein and phosphorylation of p38 mitogen-activated-protein

kinase (MAPK). Consistent with the apoptotic effects in vitro, pre-infection of T47D cells with Adl-536 or AdL540Q inhibited tumor formation when these cells were introduced into nude mice. In addition, injection of Ad1-536 and AdL540Q into pre-established T47D tumors induced tumor regression. Apoptosis, in conjunction with the activation of caspase- 3 and phosphorylation of $\mathrm{p} 38$ MAPK, was detected in the shrinking tumors. Overexpression of wild-type ER by AdwtER infection also produced antiproliferative and apoptotic effects, but to a lesser extent than the ER1-536 and L540Q mutants.

Conclusions: These results indicate that dominant negative ER mutants have the potential to induce apoptosis of T47D cells and regression of tumors. The delivery of dominant negative ERs by adenoviral vectors may provide a useful tool for targeted therapy of ER-positive breast cancer.
\end{abstract}

\section{Introduction}

Estrogens are potent stimulators of breast epithelium proliferation. The presence of estrogen receptor (ER) in breast cancer is an important prognostic factor, exceeded in predictive value only by the presence or absence of axillary lymph node metastasis (1). About two-thirds of tumors in breast cancer patients contain high levels of ER protein, and up to two-thirds of these patients respond to endocrine ablative therapy (2) or antiestrogen treatment (3-5). Other modes of disrupting ER function could provide useful new therapies for breast cancer patients.

In addition to estrogen receptor antagonists such as tamoxifen, dominant negative forms of the ER have been suggested as a method to block ER action (6-8). These include truncated receptors (ER-1-530 and ER 1-536, missing the last 65 or 59 amino acid residues), a point mutant (L540Q), and a frame shift

Send corrrespondence and reprint requests to: J. Larry Jameson, Division of Endocrinology, Metabolism and Molecular Medicine, Northwestern University Medical School, Galter Pavilion,

Suite 3-150, 251 E. Huron St., Chicago, IL, 60611-2908.

Phone: 312-926-9436; fax: 312-926-7260;

e-mail: ljameson@northwestern.edu. mutant (S554fs). Dominant negative ER mutants are transcriptionally inactive but effectively suppress wild type transcriptional activity. The formation of inactive heterodimers, competition for estrogen response element (ERE) binding, and specific transcriptional silencing have been suggested as mechanisms underlying dominant negative activity (9). Lazennec, et al. (10) demonstrated that adenovirusmediated expression of the frame-shifted ER (S554fs) suppressed the proliferation of ER-positive MCF-7 breast cancer cells. In this study, we used other dominant negative ER mutants (ER1-536 and L540Q) to examine their effect on gene transcription, cell proliferation, and apoptosis in ER-positive T47D breast cancer cells in vitro and in an in vivo animal xenograft model.

\section{Materials and Methods Generation of Transfer Plasmids and Recombinant Adenoviral Vectors}

A cassette containing the human $\operatorname{ER} \alpha(\mathrm{hER} \alpha)$ cDNA (provided by Dr. Pierre Chambon, Université Louis Pasteur, Strasbourg, France) driven by the cytomegalovirus (CMV) promoter/enhancer with a simian 
virus (SV) 40 polyadenylation $(\mathrm{p}(\mathrm{A})$ ) sequence was subcloned into an adenoviral transfer plasmid (11) based on pcDNA3 (Invitrogen, Carlsbad, CA). The dominant negative ER $\alpha$ s, 1-536hER $\alpha$ and L540QhER $\alpha$ were created using site-directed mutagenesis, and exchanged for the wild type ER $\alpha$ in the adenoviral transfer plasmid. The resulting plasmids, pCwER, pC1-536, and pCL540Q, were used to generate recombinant adenoviruses. Linearized transfer plasmids containing the expression cassette and $393 \mathrm{bp}$ of $5^{\prime}$ adenoviral sequence were ligated with ClaIdigested Ad5 309/356 DNA representing map units 3.0-100. (Ad5 309/356 is a recombinant adenovirus in which the $\mathrm{E} 3$ region is deleted. ClaI digestion removes the Ela region, resulting in a replicationdeficient virus.) The ligation products were transfected into HEK293 cells, in which cellular expression of the Ela protein allows replication of the El-deleted recombinant viruses. The purification and titration of adenoviral vectors were performed by plaque assay. Recombinant adenoviruses carrying wild-type $\mathrm{hER} \alpha$, 1-536hER $\alpha$ and L540QhER $\alpha$ were designated AdwtER, Ad1-536, and AdL540Q, respectively. AdGal, which contains $\beta$-galactosidase driven by CMV promoter, was used as a control.

An adenoviral reporter vector, AdERE-Luc, was created to investigate transcriptional activity of the wild type or dominant negative ER expressed by adenoviral vectors. The ERE2-TK109 promoter sequence was excised from ERE2-tk109-luc (12) and ligated into pGL3-promoter plasmid (Promega, Madison, WI) from which the SV40 promoter had been deleted (NheI to HindIII). Then a portion of the resulting plasmid, containing the upstream synthetic $\mathrm{p}(\mathrm{A})$ signal, two consensus EREs, a $109 \mathrm{bp}$ fragment of the thymidine kinase promoter, the firefly luciferase gene, and the downstream SV40 $\mathrm{p}(\mathrm{A})$ signal, was subcloned into the adenoviral transfer plasmid. The resulting plasmid, pC-ERELuc, was used to generate AdERE-Luc. The sequences of the expression cassettes in the adenoviral vectors were confirmed by automated DNA sequencing. Structures of the adenoviral vectors are shown in Fig. 1.

\section{Cell Culture and Infection of Recombinant Adenoviruses}

ER-positive T47D and ER-negative MDA-MB-231 breast cancer cells were provided by Dr. V. Craig Jordan (Northwestern University Medical School, Chicago, IL) and cultured in DMEM/F12 + 10\% fetal bovine serum (FBS). HEK293 embryonic kidney cells were obtained from the American Type Culture Collection (Rockville, MD) and maintained in DMEM + $10 \%$ FBS. All media were supplemented with 100 units $/ \mathrm{ml}$ penicillin and $100 \mu \mathrm{g} / \mathrm{ml}$ streptomycin; all cells were maintained at $37^{\circ} \mathrm{C}$ with $5 \% \mathrm{CO}_{2}$.

For infection of adenoviral vectors, cells were depleted of estrogen for 3 days using phenol redfree DMEM/F12 containing 5\% dextran/charcoalstripped FBS (DCC-FBS). Transduction efficiency of
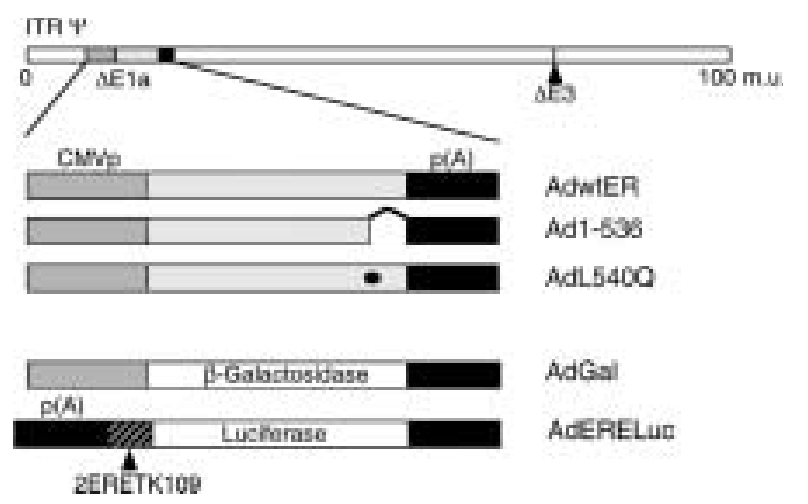

Fig. 1. Structures of recombinant adenoviruses. The adenoviral vector contains a backbone derived from adenovirus type 5 (Ad5 309/356), in which the E3 region has been deleted. The genes of interest (shaded area) were inserted in place of the El region. Five different recombinant adenoviral vectors were generated: AdwtER, Ad1-536, AdL540Q, AdGal, and AdERE-Luc. AdGal, which contains the $\beta$-galactosidase gene driven by the CMV promoter, was used to determine the efficiency of gene transduction and as a control for non-specific effects of infection. AdERE-Luc, which carries 2 estrogen response elements, the minimal thymidine kinase promoter (109 bp) (2ERE-TK 109), and the firefly luciferase gene, was used to measure ER transcriptional activity in infected cells.

adenoviral vectors in cell lines was tested using AdGal. $\beta$-galactosidase expression was detected in $95-$ to $100 \%$ of T47D cells at 48 hours after infection with AdGal at a multiplicity of infection (MOI) of 5 plaque-forming units (PFU) per cell (data not shown). MDA-MB-231 cells showed 95- to $100 \%$ expression at $10 \mathrm{PFU} / \mathrm{cell}$ (data not shown). Therefore, subsequent experiments were done using similar amounts (5 or 10 PFU/cell) of recombinant adenoviral vectors.

The transcriptional activities of ER and dominant negative ER were assayed on the 2ERE-TK109-luciferase reporter gene as described previously (12). Briefly, 12-well plates of T47D cells were infected with 1 PFU/cell AdERE-Luc and increasing amounts (1, 5, and 10 PFU/cell) of AdGal, AdwtER, Adl-536, and AdL540Q overnight. Fresh media containing E2 was added, further incubation continued for $24 \mathrm{~h}$, and then luciferase activity was assayed.

Immunofluorescence, Immunohistochemistry, and Terminal Deoxynucleotidyltransferase (TdT)-Mediated UTP End Labeling (TUNEL) Assays

To detect the expression of ER delivered by adenoviral vector, MDA-MB-231 cells were collected and mounted on slides $48 \mathrm{~h}$ after infection with adenoviral vectors. After $10 \mathrm{~min}$ air-drying, cells were fixed with ice-cold methanol followed by acetone ( 5 min each). After preincubation with serum blocking solution (ABC kit, Vector Laboratories, Inc., Burlingame, CA) for $10 \mathrm{~min}$, slides were incubated with mouse monoclonal anti-human ER $\alpha$ (1:50, D-12, sc-8005, Santa Cruz Biotechnology, Inc., Santa Cruz, CA) for $2 \mathrm{~h}$ at room temperature. After 
washing with TBS/ $0.025 \%$ Tween, staining was performed using biotinylated secondary antibodies (ABC kit, Vector Laboratories) and streptavidinFITC (1:100, Vector Laboratories).

To detect apoptosis of T47D cells infected with adenovirus, cells were collected after $6 \mathrm{~d}$ of estrogen treatment, mounted on glass slides, air-dried for $10 \mathrm{~min}$, fixed for $30 \mathrm{~min}$ in $4 \%$ paraformaldehyde, and permeabilized with buffer containing $0.1 \%$ sodium citrate and $0.4 \%$ Triton-X 100 for 2 min on ice. The cells were then washed with PBS and subjected to a modified TdT-mediated fluorescein-dUTP nick end labeling (TUNEL) reaction using the In Situ Cell Death Detection Kit, Fluorescein (Boehringer-Mannheim Co. Indianapolis, IN).

To detect the activation of p38 MAPK and caspase- 3 in T47D tumors excised from nude mice, $4 \mu \mathrm{m}$ sections of tumor in paraffin block were prepared. After deparaffinization, slides were placed in a jar containing $10 \mathrm{mM}$ sodium citrate buffer $(\mathrm{pH} 6.0)$, exposed to microwave radiation $(1000 \mathrm{~W})$ for $5 \mathrm{~min}$, and cooled to room temperature. After preincubation with serum blocking solution, slides were incubated with rabbit polyclonal anti-phospho-p38MAPK, anti-p38MAPK (1:100, New England Biolabs, Inc. Beverly, MA), and rabbit polyclonal anti-cleaved caspase-3 (1:50, Cell Signaling Technology, Inc, Beverly, MA), respectively for $1 \mathrm{~h}$ at room temperature. After washing with TBS $/ 0.025 \%$ Tween, staining was performed using $\mathrm{ABC}$ kit (Vector Laboratories). Images were obtained using a Nikon Eclipse E400 microscope (NIKON Co., Tokyo, Japan).

\section{Western Blot Analyses}

Cells were plated in $10 \mathrm{~cm}$ culture dishes at a density of $5.0 \times 10^{6}$ cells/plate. The next day, cells were infected with adenoviral vectors at a MOI of 5 PFU/cell for $5 \mathrm{~h}$. After addition of fresh media, the cells were incubated for $48 \mathrm{~h}$ with or without $1 \mathrm{nM}$ estradiol. Cells were washed twice with PBS, and whole cell lysates (ER, Bax, and Bcl-2) or nuclear protein extracts (phospho-p38 MAPK) were prepared as described previously (13). Samples containing equal amounts of protein were resolved by $10 \%$ SDS-PAGE and transferred onto nitrocellulose filters. The membranes were blocked with $5 \%$ nonfat milk in PBS for $1.5 \mathrm{~h}$ and then incubated overnight at $4^{\circ} \mathrm{C}$ with primary antibodies. For the detection of ER, mouse monoclonal anti-human ER (D-12, sc-8005, 1:400, Santa Cruz Biotechnology, Inc.) was used. Mouse monoclonal anti-Bcl-2 (1:1000, Santa Cruz Biotechnology, Inc.), and mouse monoclonal anti-Bax (1:1000, Santa Cruz Biotechnology, Inc.) were used for the detection of apoptosis regulatory proteins. Activated p38MAPK (Thr180/Tyr182-phosphorylated p38MAPK) was detected by rabbit polyclonal antiphospho-p38MAPK (1:1000, New England Biolabs).

After three washes in $0.1 \%$ Tween-20 in PBS, immunoreactive proteins were detected using an antimouse or rabbit horseradish peroxidase-conjugated antibody (1:5000, Promega, Madison, WI) and the enhanced chemiluminescence system (Amershan Pharmacia Biotech, Arlington Heights, IL). Bands were detected with Kodak (Rochester, NY) X-Omat film.

\section{Cell Proliferation Assays}

The effect of dominant negative ER on T47D cell growth was measured with a nonradioactive cell proliferation assay according to the manufacturer's protocol (Cell Titer 96 Aqueous Non-Radioactive Cell Proliferation Assay, Promega, Madison, WI). Cells were seeded in 96-well plates at a density of $5 \times 10^{3}$ cells/well and infected on the following day with adenoviral vectors at different MOIs $(0,5,10 \mathrm{PFU} / \mathrm{cell})$. Medium was replaced at $5 \mathrm{~h}$ after infection and every two days thereafter. To measure the effect of different doses of E2 (1, 10, and $100 \mathrm{nM})$, quadruplicate wells were assayed for viable cell density at day 6 . Relative density was calculated as the absorbance at $490 \mathrm{~nm}$ of the virus infected cells divided by that of the $1 \mathrm{nM} \mathrm{E2-}$ treated cells, and expressed as a percentage (mean \pm SD). In a second set of experiments, cell density was assayed at 2-day intervals over an 8 day-period with a fixed E2 concentration (1 nM).

\section{Analysis of T47D Tumors in Nude Mice}

Two different experimental designs were used. To study tumor formation, T47D cells were infected with $5 \mathrm{PFU} / \mathrm{cell}$ of adenoviruses and incubated at $37^{\circ} \mathrm{C}$ for $24 \mathrm{~h}$. Cells were collected, washed twice with PBS, resuspended in medium and injected $\left(2 \times 10^{6}\right.$ cells $)$ into the flanks of adult (8-week old) athymic female nude mice (Harlan-Sprague-Dawley, Indianapolis, IN), which had been subcutaneously implanted with 60-day estrogen pellets (Innovative Research of America, Sarasota, FL) 7 days earlier. The mice were divided into 5 different groups (6 injection sites per group): 1) no virus; 2) AdGal; 3) AdwtER; 4) Adl536; 5) AdL540Q. Animals were examined for tumor formation and growth for a period of 3 weeks.

In a second design, the effect of dominant negative ER on pre-established T47D tumors in nude mice was investigated. T47D cells $\left(1 \times 10^{7}\right)$ were injected into the flank areas of estrogen pre-implanted nude mice. Ten days after injection, tumors of about $6-7 \mathrm{~mm}$ diameter had developed and the mice were divided into 4 groups (6 tumors per group), each of which was injected with a different adenoviral vector: AdGal, AdwtER, Ad1-536, or AdL540Q. Doses of $5 \times 10^{9} \mathrm{PFU}$ were injected into the tumors on 3 successive days, from three different directions. The sizes of the tumors in all three dimensions were measured twice a week with calipers. Tumor size $\left(\mathrm{mm}^{3}\right)$ was calculated using the formula: $(3.14 \times$ length $\times$ width $\times$ depth $) / 6$. The experiment was terminated 3 weeks after injection of adenoviral vectors.

Results were subjected to analysis of variance and the statistical significance of differences between treatment groups was evaluated by Tukey's $\omega$-test. To investigate the histologic changes in 
shrinking tumors, tumors were removed from a second group of mice 7 days after injection of adenoviral vectors and embedded in paraffin. All studies involving the use of nude mice were approved by the Northwestern University Medical School Animal Care and Use Committee.

\section{Results}

\section{Expression of ER Delivered by Adenoviral Vectors}

Immunofluorescence was used to observe ER expression in ER-negative MDA-MB-231 breast cancer cells infected with adenoviral vectors. AdGal-infected cells served as a negative control. ER was detected in the nucleus of cells infected with AdwtER, Ad1-536, and AdL540Q, but not in the control cells (Fig. 2A). Using a MOI of $10 \mathrm{PFU} / \mathrm{cell}$, wt or mutant ER expression was detected in $>95 \%$ of MDA-MB-23 lcells. Similar results were obtained in ER-negative COS-7 cells (data not shown).

Western blot analyses were also performed to further characterize expression of the mutants. As expected, anti-ER immunoreactive bands of approximately $66 \mathrm{kDa}$ were detected in cells infected with AdwtER or AdL540Q. Ad1-536 infected cells showed a band of greater mobility, consistent with the reduced molecular weight of the 1-536 truncation mutant. Wild type ER and mutants were expressed at comparable levels. No ER band was detected in cells infected with AdGal (Fig. 2B). We also compared the expression levels between endogenous ER and adenovirally expressed ER in T47D cells. As assessed by Western blotting, ER levels were about 10-fold greater in T47D cells infected with 5 PFU/cell, and about 20- to 30fold greater with $10 \mathrm{PFU} / \mathrm{cell}$, than endogenous ER levels in T47D cells (data not shown).

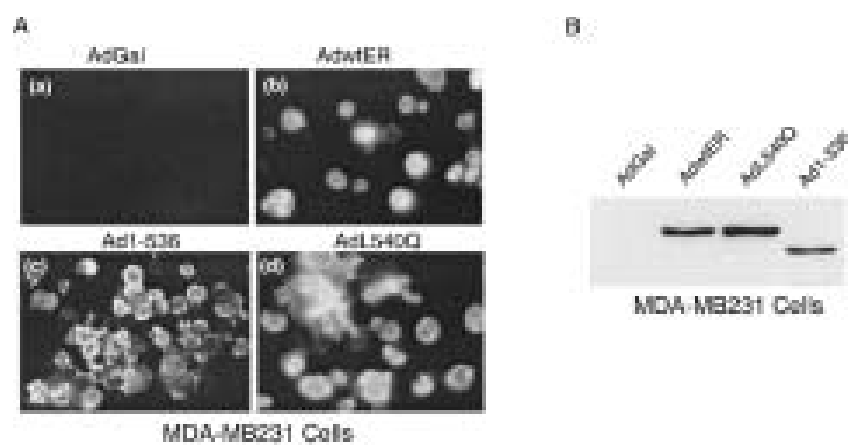

Fig. 2. Expression of the human ER $\alpha$ in MDA-MB-231 ER-negative breast carcinoma cells infected with adenoviral vectors. (A) Immunofluorescence localization of ER in MDA-MB-231 cells. MDA-MB-231 cells infected with AdGal (a), AdwtER (b), Adl-536 (c), and AdL540Q (d) were subjected to immunofluorescence staining with mouse monoclonal anti-human ER $\alpha$. (B) Western blot analysis of ER. Whole cell extracts of MDA-MB-231 cells infected with adenoviral vectors were analyzed using the same antibody. Similar results were obtained in a second independent experiment for both immunofluorescence and Western blotting.
Effect of Dominant Negative ERs on the Transcriptional Activity of the Endogenous ER in T47D Cells

To investigate whether dominant negative ER expression by adenovirus infection affects endogenous ER activation of estrogen-responsive reporter genes, T47D cells were co-infected with AdERE-Luc and adenoviruses encoding wt or dominant negative ERs. As shown in Fig. 3, E2 (1 nM) treatment stimulated ERE reporter gene activity about 20 -fold over background in control (uninfected and AdGal-infected) cells. AdwtER infection at an MOI of 1 PFU/cell increased reporter response two-fold, but higher doses of AdwtER produced no further increase. In contrast, infection with adenoviral vectors expressing dominant negative ERs suppressed the estrogen-stimulated activity in a dose-dependent manner. This suppression was greater in cells infected with Ad1-536 (91\% and $95 \%$ at 5 and $10 \mathrm{PFU} / \mathrm{cell}$ respectively) than AdL540Q (44\% and 70\%).

\section{Effect of Dominant Negative ER on T47D Cell Growth in Vitro}

To investigate whether dominant negative ERs influence cell growth, the proliferation of T47D cells was examined after infection with two different doses (5 and $10 \mathrm{PFU} / \mathrm{cell}$ ) of adenoviral vectors. As shown in Figs. 4A and B, growth of uninfected cells was stimulated by 6-day E2 treatment (1-100 nM). Adl536 or AdL540Q infection suppressed E2-stimulated

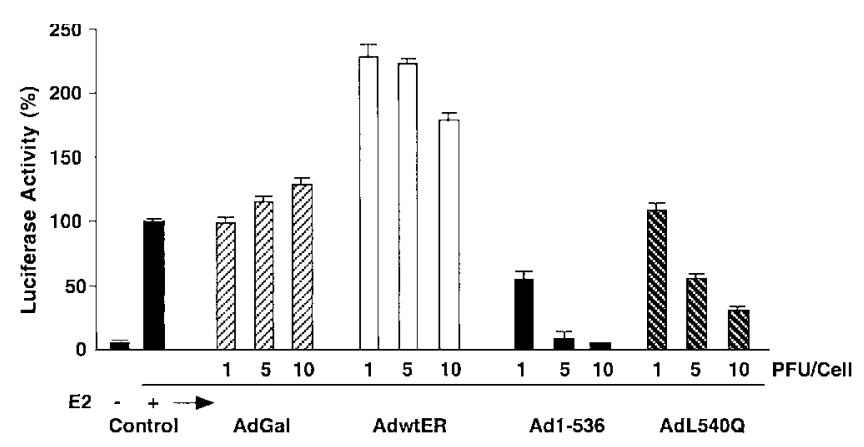

Fig. 3. Effect of dominant negative ER on transcriptional activity of the endogenous ER in T47D cells. ER transcriptional activity was assayed using the estrogenresponsive luciferase reporter vector described in Materials and Methods. Triplicate wells of T47D cells were co-infected with 1 PFU/cell AdERE-Luc and increasing amounts (1, 5, and 10 PFU/cell) of AdGal, AdwtER, Ad1-536, or AdL540Q. T47D cells infected with AdERE-Luc alone were used as a control. After overnight infection, fresh medium containing E2 (1 nM) was added, cells were incubated for an additional $24 \mathrm{~h}$, and luciferase activity was assayed. Data are expressed as a percentage (mean \pm standard deviation, $\mathrm{n}=3$ ) of luciferase activity in control T47D cells treated with E2. Reporter activity in AdL540Q-infected cells is significantly $(p<.01)$ reduced compared to Ad-Gal infected cells at MOIs of 5 and $10 \mathrm{PFU} / c e l l ;$ activity in Ad1-536-infected cells is significantly $(p<.01)$ reduced compared to AdGal- and L540Q-infected cells at all MOIs. Similar results were obtained in two additional independent experiments. 

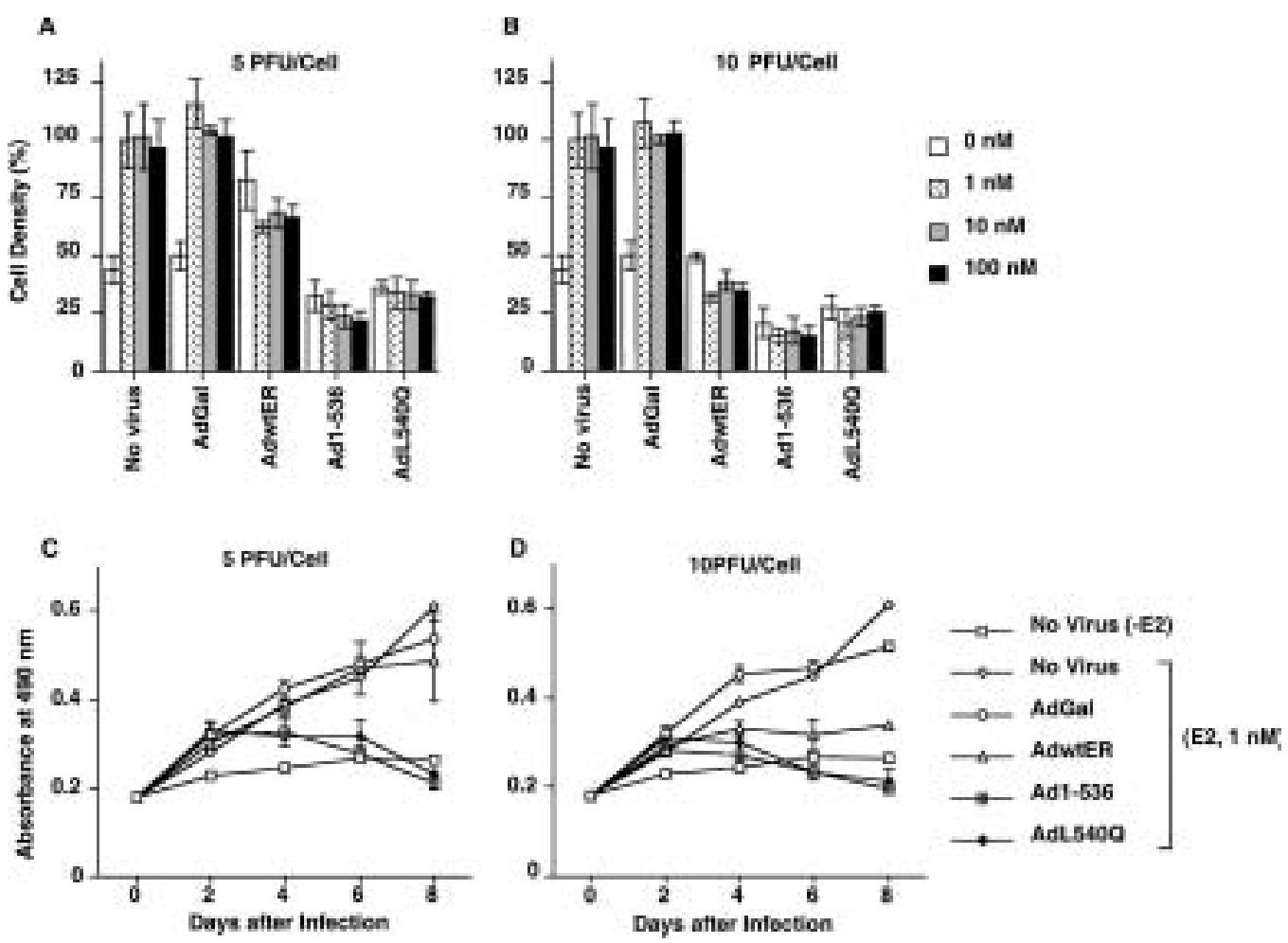

Fig. 4. Effect of dominant negative ER on the growth of T47D cells. Cells were plated, infected and treated as described in Materials and Methods. After treatment for 6 days with various doses of E2 (A, B), or at intervals over an 8-day treatment with $1 \mathrm{nM}$ E2 (C, D), cell growth was determined by a colorimetric assay. For panels A and B, cell density is expressed as a percentage, normalized to E2 treated ( $1 \mathrm{nM})$ uninfected cells. All results are plotted as means \pm standard deviations for quadruplicate wells. Similar results were obtained in two additional experiments.

growth by $60-85 \%$, depending on the mutant and viral dose. AdGal had little effect on the growth of T47D cells. Infection with AdwtER at 5 PFU/cell caused a modest increase $(\sim 50 \%)$ in growth in the absence of added E2, possibly by enhancing the effectiveness of trace levels of estrogens remaining in the stripped serum. However, in the presence of added E2, slight growth inhibition (25\% to $30 \%)$ was observed. Using a MOI 10 PFU/cell, AdwtER produced a more substantial $(\sim 70 \%)$ inhibition of E2-stimulated cell growth, and no growth increase in the absence of $\mathrm{E} 2$.

To assess the time course of these effects, T47D cells were infected with adenoviral vectors, and assayed for cell density every two days thereafter for a total of 8 days (Fig. 4C, D). Initially all cells proliferated in response to E2 (InM). Growth inhibition by Ad1-536 or AdL540Q was apparent by day 4 or 6 . AdwtER had little effect on cell growth at $5 \mathrm{PFU} / \mathrm{cell}$, while $10 \mathrm{PFU} / \mathrm{cell}$ inhibited growth more, but not as much as the dominant negative ER mutants. Growth was not affected by the AdGal virus, implying that growth inhibition was not due to nonspecific effects of viral infection. These results indicate that virally expressed dominant negative ER mutants can inhibit estrogen-stimulated growth of breast cancer cells, but several days are required for the mutant receptors to exert an inhibitory effect.

Effect of Dominant Negative ERs on Induction of Apoptosis, Bax and Bcl-2 Expression, and Activation of 338 MAPK in T47D Cells

TUNEL assays for DNA fragmentation were used to investigate whether dominant negative ERs induce apoptosis (14). A positive TUNEL reaction was obtained in T47D cells infected with Ad1-536 (51.1 \pm $6.2 \%)$ and AdL540Q $(46.1 \pm 3.4 \%)$. AdwtER infection also induced apoptosis, but the TUNEL positivity $(15.1 \pm 3.7 \%)$ was lower (Fig. 5 B, C, D; percentages represent means \pm s.d. of 4 microscope fields for each vector). The TUNEL reaction was negative in AdGal infected cells (Fig. 5A) and uninfected cells, regardless of estrogen treatment (data not shown).

Western blotting was used to examine the expression of specific proteins associated with apoptosis. T47D cells were infected with adenoviral vectors and treated with E2 for $48 \mathrm{~h}$ or $72 \mathrm{~h}$. Cells infected with AdwtER, Ad1-536, or AdL540Q showed increased expression of the pro-apoptotic Bax protein (Fig. 6A). Estrogen treatment increased expression of the anti-apoptotic protein Bcl-2 at $48 \mathrm{~h}$ and $72 \mathrm{~h}$ in uninfected cells, but infection with dominant negative ER vectors significantly decreased Bcl-2 expression. 


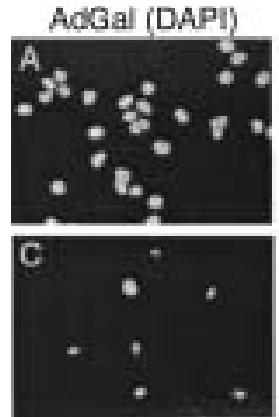

Ad1-536

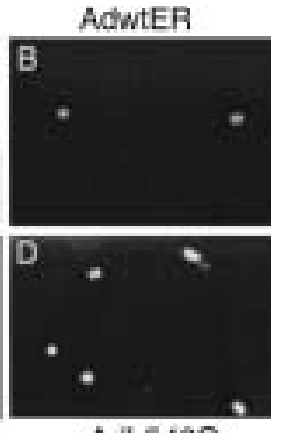

AdL5400
Fig. 5. Induction of positive TUNEL reaction. T47D cells were infected with AdGal (A), AdwtER (B), Adl-536 (C), and AdL540Q (D) at an MOI of 5 PFU/cell. After 6 days of treatment with E2 (1 nM), the TUNEL assay was performed. Fluorescence (white) indicates TUNEL labeling. AdGal-infected cells were also stained with DAPI (violet) to show cell density.

At $72 \mathrm{~h}$, a slight decrease in Bcl-2 expression was also observed in AdwtER-infected cells (Fig. 6B).

Activation of p38 MAPK kinase is associated with 4-hydroxytamoxifen-induced apoptosis in MCF-7 breast cancer cells (15). To analyze the effect dominant negative ERs on p38 MAPK activation, nuclear protein extracts were prepared from adenovirus-infected T47D cells treated with E2 over a range of times $(0,6$, 12,24 , and $48 \mathrm{~h}$ ), and extracts were analyzed by Western blotting with anti-phospho-p38 antibody (Fig 6C). Phosphorylation of p38 MAPK occurred most rapidly (by $24 \mathrm{~h}$ ) and intensely in Adl-536-infected cells. After $48 \mathrm{hr}$, phospho-p 38 was also detectable in AdL540Q-infected cells and, at much lower levels, in AdwtER-infected cells. No phospho-p38 was detected

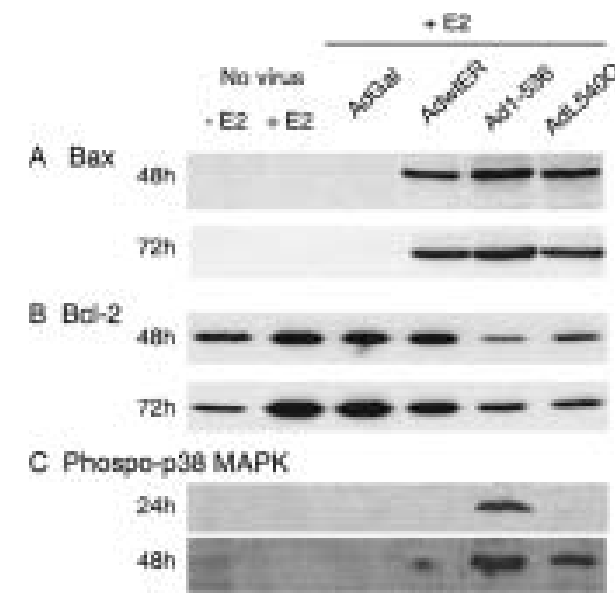

Fig. 6. Expression of Bax and Bcl-2, and activation of caspase-3 and p38 MAPK in T47D cells. Cells were infected with 5 PFU/cell of adenoviral vectors and treated with E2 over a range of times. Equal amounts of whole cell extracts (Bax, and Bcl-2) or nuclear extract (phospho-p38 MAPK) were resolved by SDS/PAGE and immunoblotted with mouse monoclonal antibodies against Bax (A), Bcl-2 (B), or phospho-p38MAPK (C). Similar results were obtained in two additional experiments. in extracts from uninfected cells or cells infected with the AdGal control vector.

\section{Effect of Dominant Negative ERs on T47D Tumors in Nude Mice}

Based on the findings that dominant negative ERs inhibit cell growth and induce apoptosis in vitro, we tested whether expression of dominant negative ERs would inhibit tumor formation by ER positive breast cancer cells in nude mice. T47D cells, either uninfected or infected with the various adenoviral expression vectors, were injected subcutaneously into estrogen-treated female athymic mice. Within 10 days, tumors formed in animals injected with uninfected cells (5 of 6 injection sites) or AdGal infected cells ( 6 of 6 sites). The tumors continued to grow and attained sizes of $44 \pm 17 \mathrm{~mm}^{3}$ and $29 \pm$ $8 \mathrm{~mm}^{3}$, respectively, 3 weeks after injection (Fig. 7A). In contrast, no tumors were detected, either by palpation or post-mortem histopathological examination,

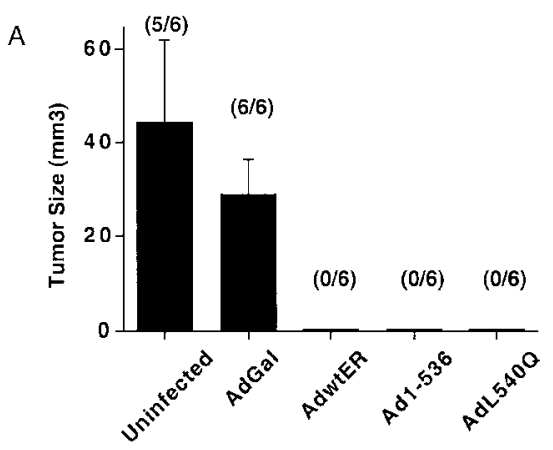

B

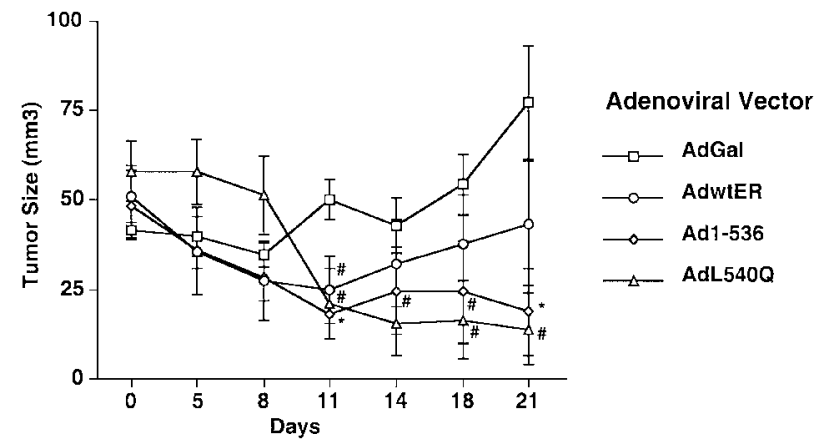

Fig. 7. Effect of dominant negative ER on T47D cell growth in vivo. (A) The effect of dominant negative ER on tumor formation by T47D cells in nude mice. T47D cells were infected with adenoviral vectors and injected into nude mice as described in Materials and Methods. Tumor volume was measured after 3 weeks. Each bar represents the mean \pm standard error (S.E.) of tumor volumes; numbers in parenthees indicate the number of tumors formed per 6 injection sites. The difference between uninfected and AdGal groups was not significant $(\mathrm{p}>.05)$. (B) Anti-tumor effect of dominant negative ER on pre-established T47D cell tumors in nude mice. Tumors were injected with adenoviral vectors as described in Materials and Methods. The tumor sizes were measured twice a week for 3 weeks. Tumor volume is presented as $\mathrm{mm}^{3}$, mean \pm S.E. for each treatment group. Points showing a statistically significant difference from AdGal are marked (\#: $\alpha$ [experimentwise error rate] $<0.05$; ${ }^{*} \alpha<0.01$ ). 
in the animals injected with cells infected with AdwtER, Ad1-536, or AdL540Q.

We also investigated the effect of dominant negative ERs introduced into pre-existing T47D tumors in nude mice. Mice were injected with uninfected cells as described above. When the tumor diameters reached about 6 to $7 \mathrm{~mm}$, each group ( $\mathrm{n}=6$ tumors) received daily intratumoral injections of adenoviral vectors for 3 days. Injection of adenoviruses encoding dominant negative ERs (Adl-536 or AdL540Q) induced tumor regression, whereas AdGal injection did not (Fig. 7B). The difference between the dominant negative ER mutants and the control vector was statistically significant ( $\alpha$ [experimentwise error rate] $<0.01$ for $1-536$ and $<0.05$ for L540Q at day 21). Although tumors injected with AdwtER showed significant growth suppression at day 11 ( $\alpha<0.05$ compared to AdGal), they regrew, and the difference between the AdwtER and control (AdGal) groups was not significant at later times.

To determine the mode of cell death, adenoviral vectors were injected into T47D cell tumors in a separate group of mice, and tumor tissue was examined 7 days later. Microscopic examination of hematoxylineosin stained tissue revealed features of apoptosis, including shrinkage of cells and condensation of nuclei, in AdwtER, Ad1-536 or AdL540Q injected tumors (Fig. 8B-D). In addition, necrosis was observed in Ad1-536 injected tumors (Fig. 8C). In contrast, AdGal-injected tumor tissue showed no such features (Fig. 8A). TUNEL assays for DNA fragmentation revealed widespread areas of apoptosis in tumors injected with AdwtER, Adl-536 or AdL540Q (Fig. $8 \mathrm{E}-\mathrm{G})$. Immunohistochemical staining showed that cleaved caspase- 3 is present in the AdwtER, Adl-536, and AdL540Q injected tumors, but not AdGal-injected controls (Fig. 8I-L). Similar results were obtained in tumor sections stained for phospho-p38 MAPK (Fig. 8M-P). Double labeling of slices from an AdL540Q-injected tumor showed that most cells that stained positive for phospho-p38 MAPK also exhibited a positive TUNEL reaction (data not shown). These histological results indicate that the tumor regression produced by injection of dominant negative ER-expressing adenoviruses is due to induction of apoptosis in the tumor cells, mediated at least in part by $\mathrm{p} 38$ MAPK and caspase-3.

\section{Discussion}

Lazennec et al. (10) demonstrated that adenovirusmediated expression of the dominant negative ER
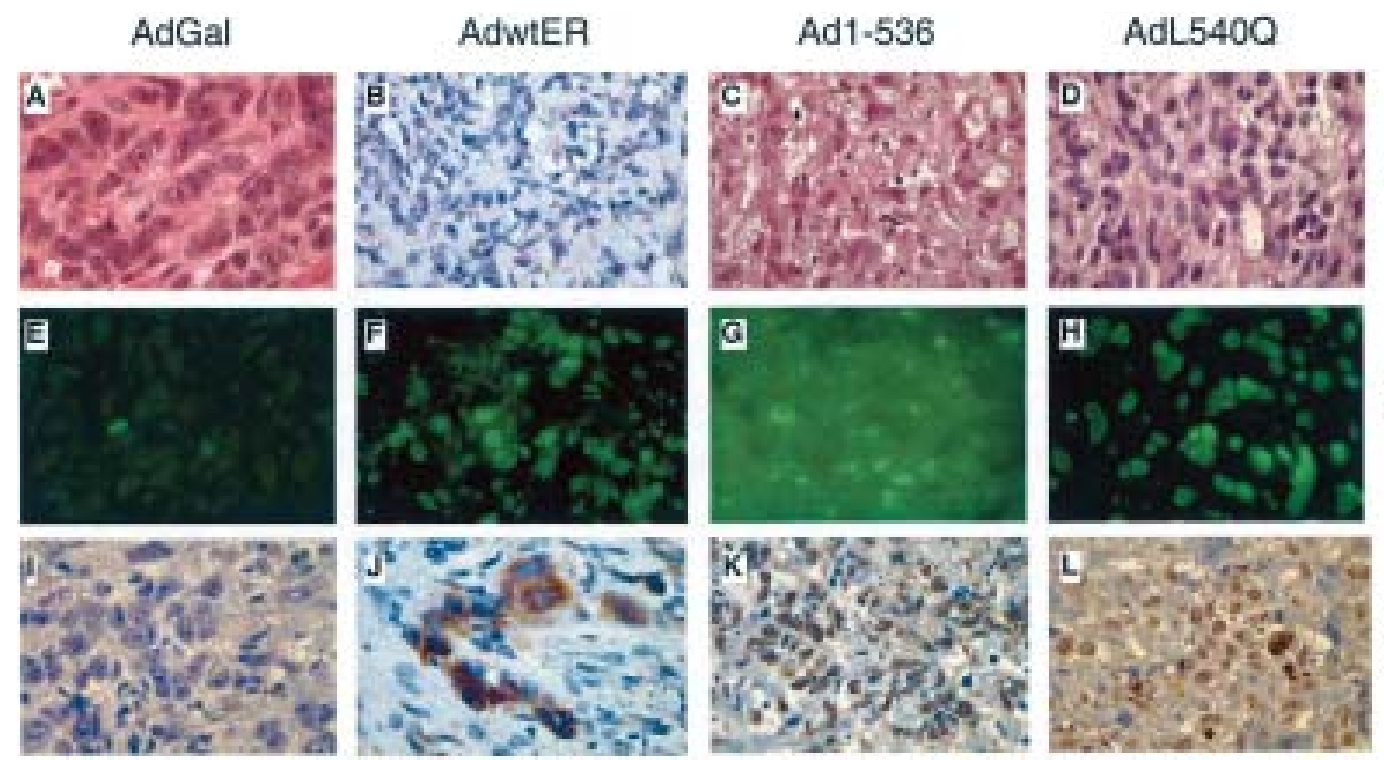

$H \& E$
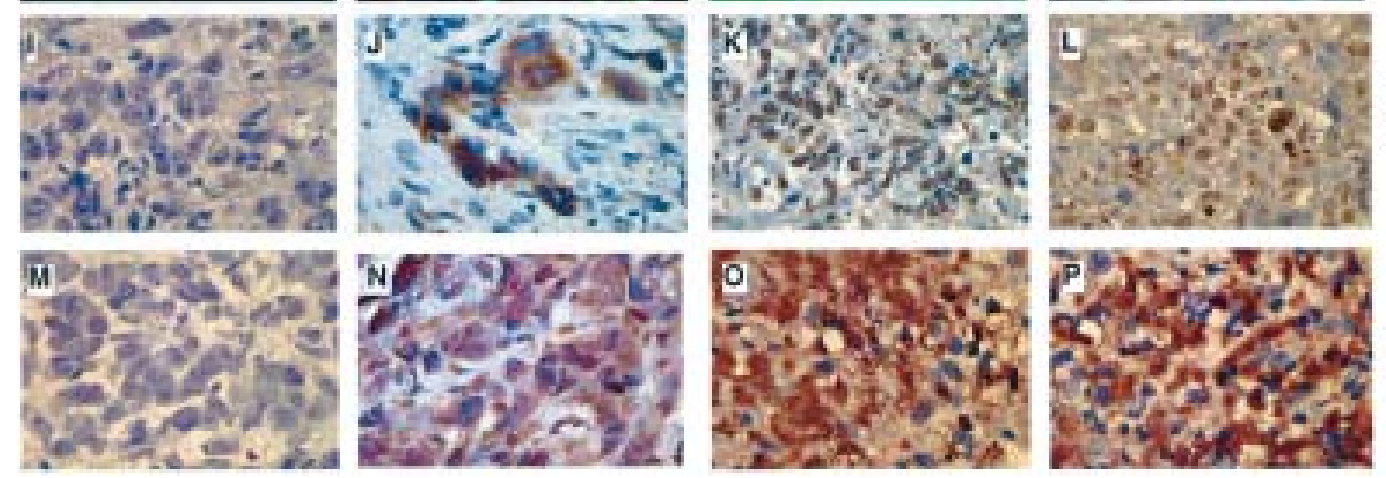

\section{Cleaved \\ Caspase-3}

Fig. 8. Apoptotic effect of dominant negative ER on T47D tumor xenografts. Seven days after injection of adenoviral vectors into T47D tumors in nude mice, tumors were removed and embedded in paraffin. Hematoxylin-eosin (HEE) staining, TUNEL assays and immunohistochemistry for cleaved caspase-3 and phospho-p38 MAPK were performed as described in Materials and Methods on tumors injected with AdGal (A, E, I, M), AdwtER (B, F, J, N), Ad1-536 (C, G, K, O) and AdL540Q (D, H, L, P). All panels are shown at $1000 \times$ magnification. (A-D): HEE stained sections. (E-H): TUNEL assay (green fluorescence indicates positive reaction). (I-L): Immunostaining for cleaved caspase-3 (brown indicates presence of antigen). (M-P): Immunostaining for phospho-p38 MAPK (brown indicates presence of antigen). Three tumors from each treatment group were sectioned and tested with similar results. 
mutant S554fs in MCF-7 cells in vitro inhibited the transcriptional activity of the endogenous ER, blocked estrogen-stimulated cell growth, and increased the proportion of cells undergoing apoptosis. In this study, we extend these observations by using two other dominant negative ERs (1-536 and L540Q) in T47D cells, another ER-positive breast cancer line. In addition, we demonstrate that infection with adenoviral vectors carrying these mutants blocks in vivo tumor formation and induces regression of established tumors.

Cells infected with wild-type ER also showed reduced growth and increased apoptosis in the presence of estrogen compared to uninfected or AdGal-infected cells. Moreover, pre-infection with AdwtER blocked tumor formation by T47D cells injected into nude mice. These results were not entirely unexpected because previous studies have shown significant growth inhibition in cells transiently or stably transfected with ER cells (16-21). In addition, Lazennec et al., examined the effect of adenovirus expressed wtER on cell proliferation in two different breast cancer cell types. In MDAMB-231 cells, which lack endogenous ER, wtER significantly inhibited proliferation in the presence of estrogen (22). In MCF-7 cells, which express a very high level of endogenous ER, inhibition was more modest and occurred only at a high MOI (10). In T47D cells, which express a lower level of endogenous ER than MCF-7 cells, we observed modest inhibition of proliferation by AdwtER at an MOI of 5 PFU/cell, and a more substantial inhibition at a higher MOI. The differences in these results are probably due to the different cell lines used and may reflect the different levels of endogenous ER expression.

Programmed cell death, or apoptosis, plays an important role in maintaining cellular homeostasis to ensure the balance between the rate of cellular proliferation and cell loss. Important genetic elements in this mechanism are Bcl-2 family proteins, including the anti-apoptotic proteins Bcl-2, Bcl-X, and Mcl-1, and the pro-apoptotic protein Bax $(23,24)$. Bax overexpression in breast cancer cells increases sensitivity to stressful stimuli, with resultant decreased cell survival and increased apoptosis (25). The ratio of Bcl-2 to Bax, rather than the absolute levels of either protein, may regulate the apoptotic response (26). Bcl-2 expression has been associated with the presence of ER in breast cancer cells (27-29). Several studies showed that patients whose tumors co-expressed $\mathrm{ER}$ and $\mathrm{Bcl}-2$ have enhanced responses to endocrine therapy $(27,30,31)$. Bcl-2 is an estrogen-regulated protein. Bcl-2 transcription is up-regulated by estrogen and down-regulated by antiestrogens in ER positive MCF-7 and T47D human breast cancer cells, whereas Bax is not (32-36). Bcl-2 up-regulation is mediated by the presence of 2 ERE sequences in the coding region of the Bcl-2 gene (37). As expected, expression of dominant negative ERs reduced the levels of Bcl-2 protein in estrogen-treated T47D cells. It also increased expression of the pro-apoptotic Bax protein. These results suggest that down-regulation of Bcl-2 and up-regulation of Bax may be involved in the induction of apoptosis by dominant negative ERs in T47D cells. Infection with AdwtER had little or no effect on Bcl-2 expression but increased Bax protein levels. However, AdwtER was less effective for inducing other markers of apoptosis (described below) and for blocking tumor formation and growth. The full role of Bax and Bcl-2 in the estrogen regulation of apoptosis in these cells remains to be determined.

Overexpression of wtER may also inhibit proliferation by non-apoptotic mechanisms. In MDA-MB231 cells, growth inhibition by adenoviral expression of wtER was correlated with decreased expression of c-myc, BRCAl and BRCA2 (22), which are protooncogenes or cancer susceptibility genes. It is also possible that high levels of ER titrate transcription factors that are necessary for cell proliferation, or induce expression of estrogen-regulated growth-inhibitory/ cytotoxic genes.

Recently, activation of the p38 MAPK pathway was demonstrated in HeLa-ER5 cells (which are stably transfected with ER) induced to undergo apoptosis by treatment with E2 or 4-hydroxytamoxifen (OHT) (15). In MCF-7 cells, which express ER endogenously, OHT also induces both p38 MAPK activation and apoptosis. In both cell types, the p38 MAPK inhibitor SB203580 protects against E2- or OHT-induced apoptosis. These results are consistent with our observations that phosphorylation of $\mathrm{p} 38$ MAPK is associated with the induction of apoptosis by dominant negative ERs and, to a lesser extent, wtER in T47D cells, both in vitro and in tumor xenografts. Activation of p38 MAPK stimulates phosphorylation and activation of p53 $(38,39)$, a protein that regulates genes involved in cell cycle progression and apoptosis (40). However, T47D cells express an inactive mutant p53 $(41,42)$, suggesting that another, still-unknown, pathway connects p38 MAPK activation and apoptosis in these cells.

Caspase-3 is a key component of the apoptotic pathway, catalyzing the proteolytic degradation of several crucial target proteins (43). Activation of caspase-3, which results from proteolytic cleavage of its inactive zymogen into active $\mathrm{p} 17$ and $\mathrm{p} 12$ subunits (44), occurs during tamoxifen-induced apoptosis of MDA-MB-231 and BT-20 cells breast cancer cells (45). In the present studies using the T47D xenograft model, activation of the caspase- 3 cascade was observed in AdwtER, Ad1-536, and AdL540Q injected tumors. These results indicate that tumor regression produced by injection of wt or dominant negative ER-expressing adenoviruses is associated with apoptosis in the tumor cells, mediated at least in part by caspase-3.

In addition to using adenovirally expressed dominant negative ERs to inhibit cell proliferation and induce apoptosis in vitro, we demonstrated their 
effectiveness in vivo, using human breast cancer xenografts in nude mice. Infection of T47D cells with dominant negative ER vectors prior to injection into the mice completely abolished tumor formation. More importantly, injection of these vectors into preestablished tumors induced regression. Histologic studies revealed apoptosis in the regressing tumors, as indicated by changes in nuclear morphology, DNA fragmentation, and activation of p38 MAPK and caspase-3. Infection with control adenovirus produced no such effects, and infection with virus encoding wtER produced only transient regression.

These results indicate that dominant negative ER mutants exert growth arrest and apoptosis in ERpositive breast cancer cells. Adenovirus-mediated delivery of these mutants may provide an alternative modality for targeted therapy of human breast cancer.

\section{Acknowledgments}

We thank Dr. Pierre Chambon for providing the cDNA of hER $\alpha$, and Dr. V. Craig Jordan for providing cells. We are grateful to Tom Kotlar for critical reading of the manuscript. This work was supported by a grant from the Northwestern Memorial Foundation, by a Center of Excellence grant from Knoll Pharmaceuticals, and National Institute Specialized Program of Research Excellence (SPORE) Grant IP50 CA89018-01. Additional support was provided by U.S. Army Medical Research and Material Command Breast Cancer Research Program grants DAMD17-94J-4082 (to JLJ) and DAMD 17-99-1-9334 (to BDG).

\section{References}

1. Harris JR, Lippman ME, Veronesi U, Willett W. (1992) Breast cancer (3). N. Engl. J. Med. 327: 473-480.

2. Pritchard KI. (1998) Ovarian ablation as adjuvant therapy for early-stage breast cancer. Cancer Treat Res. 94: 158-180.

3. Coopman P, Garcia M, Brunner N, et al. (1994) Antiproliferative and anti-estrogenic effects of ICI 164,384 and ICI 182,780 in $4-\mathrm{OH}$ tamoxifen-resistant human breastcancer cells. Int. J. Cancer 56: 295-300.

4. Kuss JT, Muss HB, Hoen H, Case LD. (1997) Tamoxifen as initial endocrine therapy for metastatic breast cancer: long term follow-up of two Piedmont Oncology Association (POA) trials. Breast Cancer Res. Treat. 42: 265-274.

5. Klijn JG, Beex LV, Mauriac L, et al. (2000) Combined treatment with buserelin and tamoxifen in premenopausal metastatic breast cancer: a randomized study [see comments]. J. Natl. Cancer Inst. 92: 903-911.

6. Ince BA, Zhuang Y, Wrenn CK, et al. (1993) Powerful dominant negative mutants of the human estrogen receptor. J. Biol. Chem. 268: 14026-14032.

7. Ince BA, Schodin DJ, Shapiro DJ, Katzenellenbogen BS. (1995) Repression of endogenous estrogen receptor activity in MCF-7 human breast cancer cells by dominant negative estrogen receptors. Endocrinology 136: 3194-3199.

8. Chien PY, Ito M, Park Y, et al. (1999) A fusion protein of the estrogen receptor (ER) and nuclear receptor corepressor (NCoR) strongly inhibits estrogen-dependent responses in breast cancer cells. Mol. Endocrinol. 13: 2122-2136.

9. Schodin DJ, Zhuang Y, Shapiro DJ, Katzenellenbogen BS. (1995) Analysis of mechanisms that determine dominant negative estrogen receptor effectiveness. J. Biol. Chem. 270: 31163-31171.
10. Lazennec G, Alcorn JL, Katzenellenbogen BS. (1999) Adenovirus-mediated delivery of a dominant negative estrogen receptor gene abrogates estrogen-stimulated gene expression and breast cancer cell proliferation. Mol. Endocrinol. 13: 969-980.

11. Lee EJ, Anderson LM, Thimmapaya B, Jameson JL. (1999) Targeted expression of toxic genes directed by pituitary hormone promoters: a potential strategy for adenovirusmediated gene therapy of pituitary tumors. J. Clin. Endocrinol. Metab. 84: 786-794.

12. Gehm BD, McAndrews JM, Jordan VC, Jameson JL. (2000) EGF activates highly selective estrogen-responsive reporter plasmids by an ER-independent pathway. Mol. Cell. Endocrinol. 159: 53-62.

13. Lee EJ, Duan WD, Kotlar T, Jameson JL. (2001) Restoration of growth hormone-releasing hormone (GHRH) responsiveness in pituitary GH3 cells by adenovirus-directed expression of the human GHRH receptor. Endocrinology 142: in press.

14. Su ZZ, Madireddi MT, Lin JJ, et al. (1998) The cancer growth suppressor gene mda-7 selectively induces apoptosis in human breast cancer cells and inhibits tumor growth in nude mice. Proc. Natl. Acad. Sci. USA 95: 14400-14405.

15. Zhang CC, Shapiro DJ. (2000) Activation of the p38 mitogenactivated protein kinase pathway by estrogen or by 4hydroxytamoxifen is coupled to estrogen receptor-induced apoptosis. J. Biol. Chem. 275: 479-486.

16. Kushner PJ, Hort E, Shine J, et al. (1990) Construction of cell lines that express high levels of the human estrogen receptor and are killed by estrogens. Mol. Endocrinol. 4: 1465-1473.

17. Maminta ML, Molteni A, Rosen ST. (1991) Stable expression of the human estrogen receptor in HeLa cells by infection: Effect of estrogen on cell proliferation and c-myc expression. Mol. Cell. Endocrinol. 78: 61-69.

18. Jiang SY, Jordan VC. (1992) Growth regulation of estrogen receptor-negative breast cancer cells transfected with complementary DNAs for estrogen receptor. J. Natl. Cancer Inst. 84: 580591.

19. Lee Y, Renaud RA, Friedrich TC, Gorski J. (1998) Estrogen causes cell death of estrogen receptor stably transfected cells via apoptosis. J. Steroid Biochem. Mol. Biol. 67: 327-332.

20. Kousteni S, Bellido T, Plotkin LI, et al. (2001) Nongenotropic, sex-nonspecific signaling through the estrogen or androgen receptors: dissociation from transcriptional activity. Cell 104: 719-730.

21. Lee EJ, Duan WR, Jakacka M, et al. (2001) Dominant negative ER induces apoptosis in $\mathrm{GH}(4)$ pituitary lactotrope cells and inhibits tumor growth in nude mice. Endocrinology 142: 3756-3763.

22. Lazennec G, Katzenellenbogen BS. (1999) Expression of human estrogen receptor using an efficient adenoviral gene delivery system is able to restore hormone-dependent features to estrogen receptor-negative breast carcinoma cells. Mol. Cell. Endocrinol. 149: 93-105.

23. Raff MC. (1992) Social controls on cell survival and cell death. Nature 356: 397-400.

24. White E. (1996) Life, death, and the pursuit of apoptosis. Genes Dev. 10: 1-15.

25. Sakakura C, Sweeney EA, Shirahama T, et al. (1996) Overexpression of bax sensitizes human breast cancer MCF-7 cells to radiation-induced apoptosis. Int. J. Cancer 67: 101-105.

26. Butt AJ, Firth SM, King MA, Baxter RC. (2000) Insulin-like growth factor-binding protein-3 modulates expression of Bax and Bcl-2 and potentiates p53-independent radiation-induced apoptosis in human breast cancer cells. J Biol Chem. 275(50): 39174-39181.

27. Gee JM, Robertson JF, Ellis IO, et al. (1994) Immunocytochemical localization of BCL-2 protein in human breast cancers and its relationship to a series of prognostic markers and response to endocrine therapy. Int. J. Cancer 59: 619-628.

28. Leek RD, Kaklamanis L, Pezzella F, et al. (1994) bcl-2 in normal human breast and carcinoma, association with oestrogen receptor-positive, epidermal growth factor receptor-negative tumours and in situ cancer. Br. J. Cancer 69: 135-139. 
29. Yang Q, Sakurai T, Jing X, et al. (1999) Expression of Bcl-2, but not Bax, correlates with estrogen receptor status and tumor proliferation in invasive breast carcinoma. Pathol. Int. 49: 775-780.

30. Elledge RM, Green S, Howes L, et al. (1997) bcl-2, p53, and response to tamoxifen in estrogen receptor-positive metastatic breast cancer: a Southwest Oncology Group study. J. Clin. Oncol. 15: 1916-1922.

31. Keen JC, Dixon JM, Miller EP, et al. (1997) The expression of Ki-S 1 and BCL-2 and the response to primary tamoxifen therapy in elderly patients with breast cancer. Breast Cancer Res. Treat 44: 123-133.

32. Teixeira C, Reed JC, Pratt MA. (1995) Estrogen promotes chemotherapeutic drug resistance by a mechanism involving $\mathrm{Bcl}-2$ proto-oncogene expression in human breast cancer cells. Cancer Res. 55: 3902-3907.

33. Wang TT, Phang JM. (1995) Effects of estrogen on apoptotic pathways in human breast cancer cell line MCF-7. Cancer Res. 55: $2487-2489$.

34. Lapointe J, Fournier A, Richard V, Labrie C. (1999) Androgens down-regulate bcl-2 protooncogene expression in ZR-75-1 human breast cancer cells. Endocrinology 140: 416-42 1.

35. Dong L, Wang W, Wang F, et al. (1999) Mechanisms of transcriptional activation of bcl-2 gene expression by 17 betaestradiol in breast cancer cells. J. Biol. Chem. 274: 32099-32 107.

36. Zhang GJ, Kimijima I, Onda M, et al. (1999) Tamoxifeninduced apoptosis in breast cancer cells relates to downregulation of bcl-2, but not bax and bcl-X(L), without alteration of p53 protein levels. Clin. Cancer Res. 5: 2971-2977.

37. Perillo B, Sasso A, Abbondanza C, Palumbo G. (2000) 17beta-estradiol inhibits apoptosis in MCF-7 cells, inducing bcl-2 expression via two estrogen-responsive elements present in the coding sequence. Mol. Cell Biol. 20: 2890-2901.

38. Huang C, Ma WY, Maxiner A, et al. (1999) p38 kinase mediates UV-induced phosphorylation of $\mathrm{p} 53$ protein at serine 389. J. Biol. Chem. 274: 12229-12235.

39. Sanchez-Prieto R, Rojas JM, Taya Y, Gutkind JS. (2000) A role for the p38 mitogen-acitvated protein kinase pathway in the transcriptional activation of $\mathrm{p} 53$ on genotoxic stress by chemotherapeutic agents. Cancer Res. 60: 2464-2472.

40. Kobayashi T, Ruan S, Jabbur JR, et al. (1998) Differential p53 phosphorylation and activation of apoptosis-promoting genes Bax and Fas/APO-1 by irradiation and ara-C treatment. Cell Death Differ. 5: 584-591.

41. Nigro JM, Baker SJ, Preisinger AC, et al. (1989) Mutations in the p53 gene occur in diverse human tumour types. Nature 342: 705-708.

42. Bartek J, Iggo R, Gannon J, Lane DP. (1990) Genetic and immunochemical analysis of mutant p53 in human breast cancer cell lines. Oncogene 5: 893-899.

43. Fernandes-Alnemri T, Litwack G, Alnemri ES. (1994) CPP32, a novel human apoptotic protein with homology to Caenorhabditis elegans cell death protein Ced-3 and mammalian interleukin-1 beta-converting enzyme. J. Biol. Chem. 269: 30761-30764.

44. Nicholson DW, Ali A, Thornberry NA, et al. (1995) Identification and inhibition of the ICE/CED-3 protease necessary for mammalian apoptosis. Nature 376: 37-43.

45. Mandlekar S, Yu R, Tan TH, Kong AN. (2000) Activation of caspase-3 and c-Jun NH2-terminal kinase-1 signaling pathways in tamoxifen-induced apoptosis of human breast cancer cells. Cancer Res. 60: 5995-6000. 